\title{
A recruitment method to obtain community samples of children for survey research in Switzerland
}

\section{Journal Article}

\section{Author(s):}

Mohler-Kuo, Meichun; Jann, Ben; Dey, Michelle; Zellweger, Ueli

Publication date:

2011

Permanent link:

https://doi.org/10.3929/ethz-b-000160316

Rights / license:

In Copyright - Non-Commercial Use Permitted

\section{Originally published in:}

International Journal of Public Health 56(3), https://doi.org/10.1007/s00038-011-0250-z 


\title{
A recruitment method to obtain community samples of children for survey research in Switzerland
}

\author{
Meichun Mohler-Kuo • Ben Jann • Michelle Dey • \\ Ueli Zellweger
}

Received: 24 August 2010/Revised: 15 March 2011/Accepted: 24 March 2011/Published online: 19 April 2011

(C) Swiss School of Public Health 2011

\section{Introduction}

In conducting epidemiological surveys of children in Switzerland, it is often very difficult to recruit large, representative community samples. To date, most large epidemiological surveys on children have used convenience samples, like school children (Jeannin et al. 2005); (Kuntsche and Kuendig 2006); (Steinhausen et al. 1998). One major limitation of school surveys is that certain information about the family and parents is often unavailable.

Unlike other countries such as the USA, Switzerland has no so-called 'census tracts' (geographic regions defined for the purpose of taking a census) that can be used as sampling units. Most Swiss epidemiological surveys of adults have adopted two-stage sampling, selecting households by address or telephone number. However, information on household structure is not available before contacting the families. After calling a particular number or selecting a certain address, a designated person, usually of a particular age and/or gender, is chosen to participate. However, this method is impractical for studies on children, due to the narrow age range. When we only want to sample children, and especially children of a particular age (e.g. those 10-14 years old), we must screen more households to achieve a desired sample size.

This Hints \& Kinks presents a method for recruiting random community samples of children through local

M. Mohler-Kuo $(\bowtie) \cdot$ M. Dey · U. Zellweger Institute of Social and Preventive Medicine, niversity of Zurich, Hirschengraben 84, 8001 Zurich, Switzerland

e-mail: meichun.mohler-kuo@uzh.ch

B. Jann

Institute of Sociology, University of Bern, Bern, Switzerland political municipalities (German: Gemeinden/French: communes/Italian: comuni) in Switzerland. Municipalities are the smallest governmental division in Switzerland. Their number has gradually decreased (from 3,095 in 1960 to 2,596 in 2010), mainly because smaller municipalities have found it difficult to absorb the necessary administrative costs of civil services. The population size of a municipality varies from less than 20 to more than 3,00,000 for a city like Zurich (Bundesamt für Statistik 2010).

Every resident in Switzerland, regardless of nationality, must register in their municipality; consequently, municipalities have the most complete socio-demographic information and could be used as a basic sampling unit. We conducted a pilot study in June 2007 at Canton of Zurich to test this method of selecting random samples through municipalities. The underlying purpose of the study was to estimate the prevalence of Swiss children with special healthcare needs.

\section{Methods}

Sampling

Our goal was to screen a representative sample of 3,500 children of 10-15 years (year of birth: 1993-1997) in the Canton of Zurich. Two-stage sampling was used to select the sample. During the first stage, using the Bernese sampling plan (Jann 2007), we selected 35 out of a total of 171 municipalities to participate. The basic principle of the Bernese sampling plan is to divide the population according to the size of municipalities, and perform simple random sampling in large municipalities (like Zurich, Winterthur, and Uster), each as its own stratum. Conversely, in small municipalities, a cluster sample is 
drawn (using probability-proportional-to-size sampling of municipalities, in each of which a fixed number of children was selected). Using such partial clustering makes it possible to limit the number of municipalities in the sample without sacrificing as much statistical efficiency as with a typical cluster sample. Note that the Bernese sampling plan is a equal probability of selection method (EPSEM) and hence yields representative target samples (see Jann (2007) and Appendix for further details).

During the second stage, we asked each selected municipality to draw a random sample of children who met inclusion criteria. Depending upon the size of the municipality, between 79 and 707 children were sampled (an exception being 39 and 40 children from two small communes that were combined into a single cluster).

Procedures

Before asking municipalities to participate, we needed to obtain permission to release individual data from the cantonal and/or city data protection committee (Datenschutzenkommission). After we received this permission, we sent an invitation letter to selected municipalities, explaining the study and its purpose, and a letter of permission from the Data Protection Committee. We asked each selected municipality to draw a random sample of children born between 1993 and 1997. In addition, we asked for detailed demographic information about each child (last and first name, birth date, gender, address, nationality) and their parents (last and first name). Telephone numbers were crucial to our study, but unavailable from the municipalities; hence, we had to seek them ourselves after we had received all the information from municipalities that was available. If a municipality failed to respond within a month, a followup telephone call was made to encourage participation.

Telephone number search

Once all the information regarding the sample was received from the municipalities, we started to search for corresponding telephone numbers using Swiss telephone directories published on the internet (http://www.tel. search.ch) The telephone numbers were then sent to the CATI center to conduct screening with parents via telephone interviews. To those families without an identifiable or valid telephone number, a written questionnaire was sent.

\section{Results}

As shown in Fig. 1, among the 35 selected municipalities, 33 (collaboration rate 94\%) provided names and addresses, resulting in 3,341 randomly selected children. We further

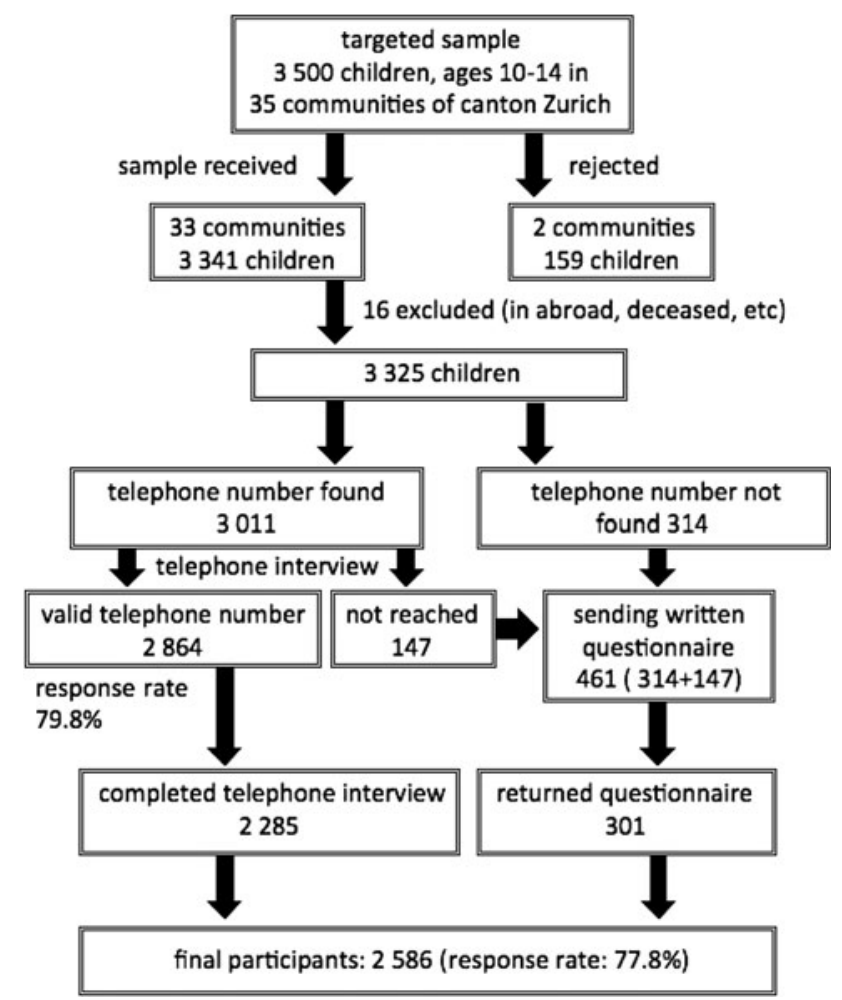

Fig. 1 Sample recruitment and screening illustration of sample recruitment and screening. 2007 Zurich Study of Children with Special Health Care Needs

excluded 16 children from the sample because they were abroad, deceased or relocated out of the municipality, leaving 3,325 children available for screening. However, some municipalities did not provide all the requested information due to different regulations of data protection. Lack of certain information such as parent's names could make telephone searching more difficult. Initially, telephone numbers for 3,011 children were found through published telephone directories, among which 2,864 were valid. A total of 2,285 families were screened by telephone interview (response rate $79.8 \%$ ). We sent a written questionnaire to 461 families (147 and 314 families whose telephone numbers were not found or invalid, respectively), 301 families returned these questionnaires (response rate $65.3 \%$ ). Altogether, 2,587 eligible families (77.8\%) participated in the study.

The demographic characteristics of the sample of 3,341 children originally received from the 33 municipalities were quite similar to the total population in terms of the distribution of sex, age, and the nationality as shown in Table 1. However, among final participants, children of non-Swiss families are slightly underrepresented due to the language barriers. This is a well-recognized problem in most of the surveys. 
Table 1 Demographic comparison between total population and recruited sample and final participants

\begin{tabular}{llll}
\hline & $\begin{array}{l}\text { Total population in } \\
\text { Canton Zurich }\end{array}$ & $\begin{array}{l}\text { Sample received } \\
\text { from 33 municipalities } \\
N=64,144(\%)\end{array}$ & $\begin{array}{l}\text { Final } \\
\text { participants } \\
N=2,586(\%)\end{array}$ \\
\hline Sex & & & \\
Male & 51.4 & 53.2 & 53.1 \\
Female & 48.6 & 46.8 & 46.9 \\
Age & & & \\
10 & 19.8 & 19.1 & 18.7 \\
11 & 20.1 & 20.4 & 20.6 \\
12 & 19.9 & 20.4 & 20.7 \\
13 & 20.0 & 21.4 & 21.5 \\
14 & 20.1 & 18.7 & 18.5 \\
Nationality & & & 82.0 \\
Swiss & 77.8 & 76.6 & 18.0 \\
Non-Swiss & 22.2 & 23.4 & \\
\hline
\end{tabular}

Demographic comparison of population and participants. 2007 Zurich Study of Children with Special Health Care Needs

a Total population in 2007

b Nationality was not available for 432 of the 3,341 children received from municipalities and for 376 of the 2,586 children in final participants the target sample size (the number of children to be sampled). Assuming that a list of municipalities is available and for each municipality $\mathrm{j}$ the number of population members $N_{\mathrm{j}}$ is known. The goal of the Bernese Sampling Plan is to draw a sample such that the number of municipalities in the sample is limited or, more specifically, that a minimum number of at least $k$ sample members is allotted to each municipality in the sample. In a simple random sample (SRS) the number of expected sample members in municipality $\mathrm{j}$ is equal to $P \times N_{\mathrm{j}}$, with $p=n / N$, which may reach the critical $k$ in large municipalities. Hence, the Bernese Sampling Plan starts off by dividing the municipalities into two groups, large municipalities for which $p \times N_{\mathrm{j}} \geq k$ and small municipalities with $p \times N_{\mathrm{j}}<k$. Let $N^{\text {small }}$ denote the total size of the population living in the small municipalities. Then, in each of the large municipalities, an SRS of $n_{\mathrm{j}}=p \times N_{\mathrm{j}}$ subjects (with random rounding for non-integer $n_{\mathrm{j}}$ ) can be drawn. Among the small municipalities, however, $p / k \times N^{\text {small }}$ clusters of size $k$ are selected by sampling municipalities proportional to size PPS and, within each sampled municipality, drawing an SRS of $k$ subjects. As shown by Jann (2007), the depicted procedure is an EPSEM with an a priori sampling probability of $p=n / N$ for each population member.

\section{References}

Bundesamt für Statistik (2010) Schweiz - die Gemeinde. Institutionelle Gliederung der Schweiz

Jann B (2007) Überlegungen zum Berner Stichprobenplan. Swiss J Sociol 33:307-325

Jeannin A, Narring F, Tschumper A, Bonivento LI, Addor V, Butikofer A, Suris JC, Diserens C, Alsaker F, van Melle G, Michaud PA (2005) Self-reported health needs and use of primary health care services by adolescents enrolled in post-mandatory schools or
Let $N$ be the size of the population (e.g. the number of children of ages 10-15 in the Canton of Zurich) and $n$ be 
vocational training programmes in Switzerland. Swiss Med Wkly 135:11-18

Kuntsche EN, Kuendig H (2006) What is worse? A hierarchy of family-related risk factors predicting alcohol use in adolescence. Subst Use Misuse 41:71-86
Steinhausen HC, Winkler C, Meier MM, Kannenberg R (1998) Prevalence of child and adolescent psychiatric disorders: the Zurich epidemiological study. Acta Psychiat Scand 98:262-271 